\title{
Laparoscopic treatment of a huge seminal vesicle calculus: A case report and review of literature
}

\author{
HAN YANG, YINLEI WANG, JIE GAO, PING WANG, XINGKANG JIANG, DAWEI TIAN and HAILONG HU \\ Department of Urology, The Second Hospital of Tianjin Medical University, Tianjin 300211, P.R. China
}

Received January 25, 2019; Accepted September 10, 2019

DOI: $10.3892 /$ etm.2019.8160

\begin{abstract}
A 67-year-old male patient had a history of repeated urinary tract infections for numerous years. X-ray examination revealed a large calcific density in the pelvic cavity, with a diameter of $10.4 \mathrm{~cm}$ and a CT value of $792.9 \mathrm{HU}$. On MRI of the pelvis, the lesion displayed with extremely low signals. The inside of the stone had a concentric ring shape with a slightly higher signal and the patient was diagnosed with a large left seminal vesicle calculus. Laparoscopic surgery was selected to treat the stone. The patient recovered rapidly and the symptoms, including urgency, urinary frequency, as well as lower abdominal and perineal pain, were obviously improved. The present case study reports on the largest seminal vesicle calculus reported in the current English literature in addition to a brief literature review. Cases of seminal vesicle calculi (SVC) are rare. The present study reports on a case of SVC, which is the largest reported in current English literature, to the best of our knowledge.
\end{abstract}

\section{Case report}

A 67-year-old male had been suffering from recurrent lower urinary tract infections for 5 years. The major manifestations were urgency and urinary frequency, lower abdominal and perineal pain, and occasionally hematuria. The patient's self-administered antibiotics improved his symptoms, but these symptoms often occured repeatedly. The patient also previously had hypospadias, and $>40$ years ago, he had received hypospadias repair and cystostomy. The patient had not received any treatment for his left cryptorchidism, azoospermia or infertility.

On digital rectal examination, prostate palpation was not obvious, a stony hard mass was present at the distal end and it was not possible to assess the boundaries. Scars from the previous surgery for hypospadias and vesicostomy were

Correspondence to: Dr Hailong Hu, Department of Urology, The Second Hospital of Tianjin Medical University, 23 Pingjiang Road, Hexi, Tianjin 300211, P.R. China

E-mail: hhllove2004@163.com

Key words: calculi, hypospadias, laparoscopic, seminal vesicles present. The right testicle was atrophic and had an empty left hemiscrotum. No abnormalities were observed in body temperature on admission, routine blood tests, biochemical tests and electrocardiogram. Parathyroid hormone levels, prostate-specific antigen, coagulation function, androgen levels and cytological results, as well as renal and liver function, were normal and hepatitis tests were negative. The prostate-specific antigen level was $0.01 \mathrm{ng} / \mathrm{ml}$. The patient's blood type was $\mathrm{B}$ and $\mathrm{RH}$ positive. Urinalysis revealed a leukocyte count of 12.83 per high-power field (HPF), an erythrocyte count of 7.09 per HPF and a squamous cell count of 12.66 per HPF. The urine culture results indicated an Escherichia coli infection. An X-ray of the pelvis displayed a large calcific density in the pelvic cavity (Fig. 1). CT revealed a large round dense shadow with a clear boundary that appeared between the bladder and rectum, which was $10.4 \mathrm{~cm}$ in diameter and a $\mathrm{CT}$ value of $\sim 792.9$ HU. The bladder, rectum and sigmoid colon were obviously compressed and displaced (Fig. 2). MRI of the pelvis indicated a large abnormal signal that was visible between the bladder and the rectum, and the lesion was dominated by extremely low signals. The inside of the stone appeared as a concentric ring shape with a slightly higher signal (Fig. 3).

The urinary infection was controlled using suitable antibiotics. An endoscopic examination was performed before the removal of the calculus to further confirm the diagnosis of SVC. Due to urethral stricture, urethral dilatation was performed intra-operatively by using a 24 French (Fr) urethral sound, and subsequently, a 8.5/9.8 Fr rigid ureteroscope was successfully placed under guidance. It was observed that the anterior hypospadias repair had healed to leave a stenosis scar and the seminal hillock was obviously expanded. However, it was not possible to place the ureteroscope into the seminal vesicle around the giant calculus. The diagnosis of SVC was further confirmed.

Considering the size of the calculus, laparoscopic surgery was selected for removal of the calculus. The easily identifiable vas deferens was used as a guide for seminal vesicle resection (Fig. 4). It was observed that the left seminal vesicle was significantly enlarged. The bottom of the seminal vesicle was dissected by using an ultrasonic knife and the calculus was then removed and placed in a specimen bag. The inner wall of the seminal vesicle was smooth and the opening of the seminal vesicle was clearly visible. The dilated seminal vesicle wall was dissected and almost completely excised. Serious adhesions were observed between the seminal 


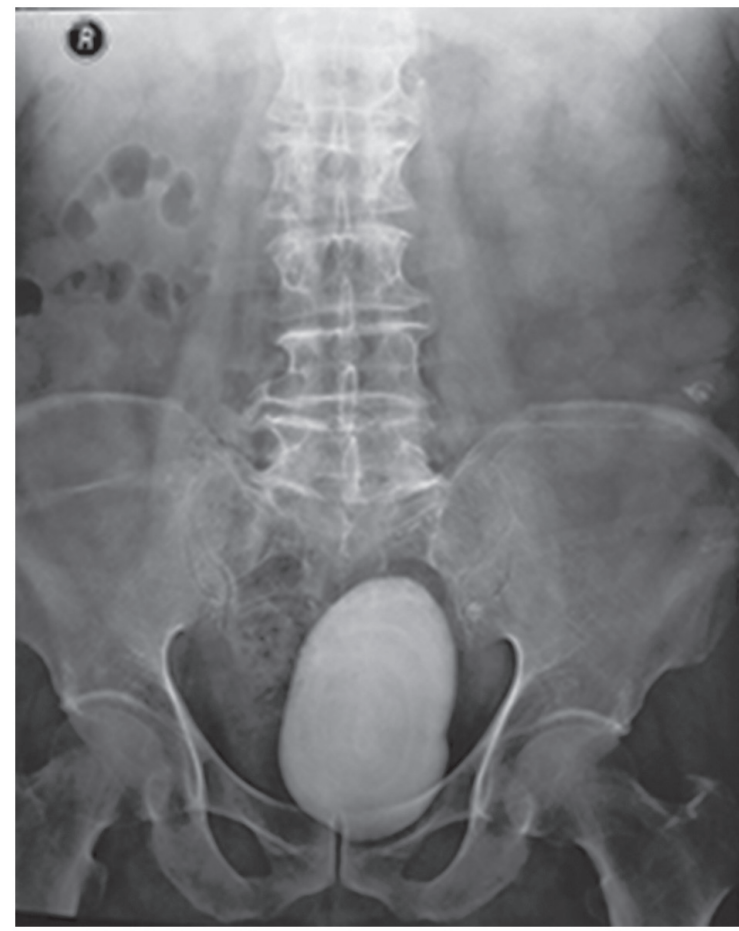

Figure 1. Pelvis X-ray showing a large calcific density in the pelvic cavity.

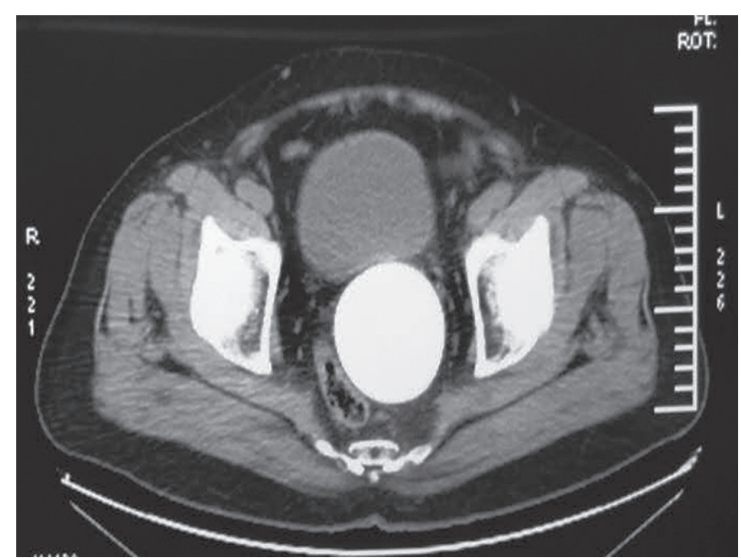

Figure 2. CT of the pelvis showing a large round dense shadow with clear boundary appeared between the bladder and rectum.

vesicle wall and the pelvic tissue. The remaining vesicle was closed with a continuous locking suture. Upon examination of the left groin area, the inguinal hernia was not obvious and no cryptorchid tissue was observed. The calculus and dilated wall were removed through the expanded umbilical incision. The calculus was light brown-colored, had a round and smooth appearance, measured roughly $10.4 \times 6.0 \times 6.9 \mathrm{~cm}$ (Fig. 5). Pathological analysis indicated that the cystic wall consisted of proliferating fiber smooth muscle vascular tissue. The covered mucosa was a proliferating squamous epithelium with hyperkeratosis with massive chronic inflammatory cell infiltration.

The patient recovered well without any complaints or complications. The transurethral catheter was removed after 3 days and was discharged on post-operative day 5 . The patient
A

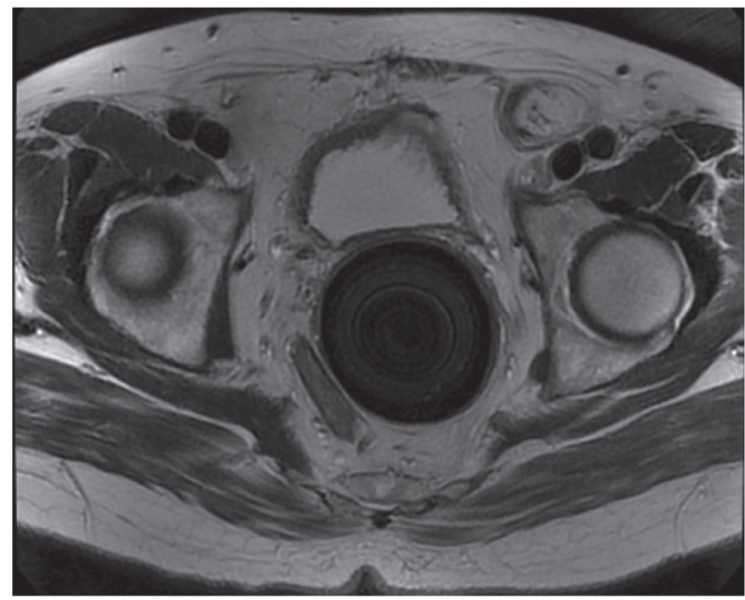

B

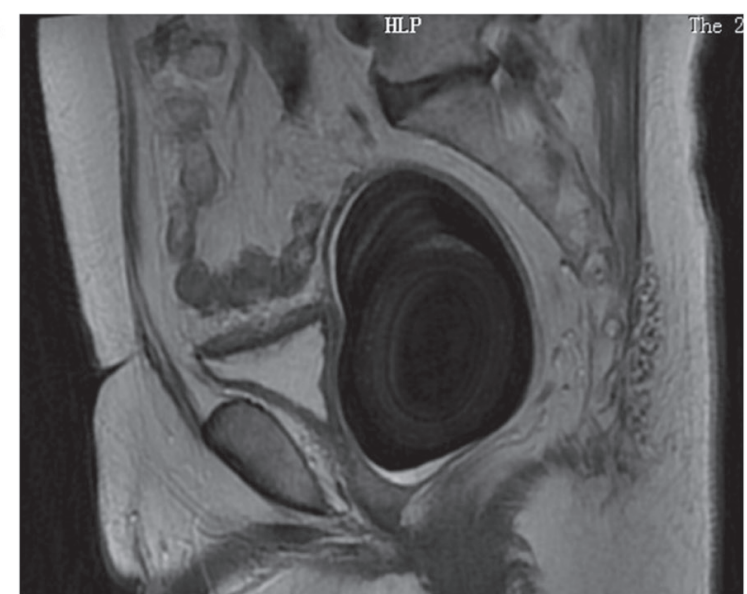

C

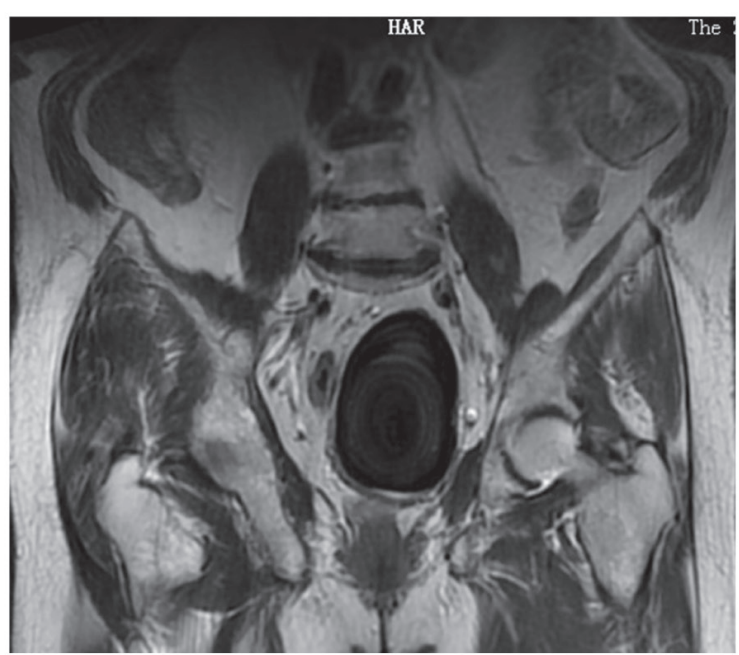

Figure 3. MRI of the pelvis showing a large hypointense abnormality. (A) The rectum and bladder were deformed and displaced. (B) Sagittal view showing the stone occupying most of the pelvic space. (C) The stone was compressing the rectum.

was regularly followed up and the symptoms were obviously improved compared with those prior to the surgery.

\section{Discussion}

The first case of SVC was reported in 1928, and since then, only a few clinically reported cases have been described (1). The definite mechanisms of the formation of SVC remain elusive; however, it usually occurs in patients with infection, urinary 


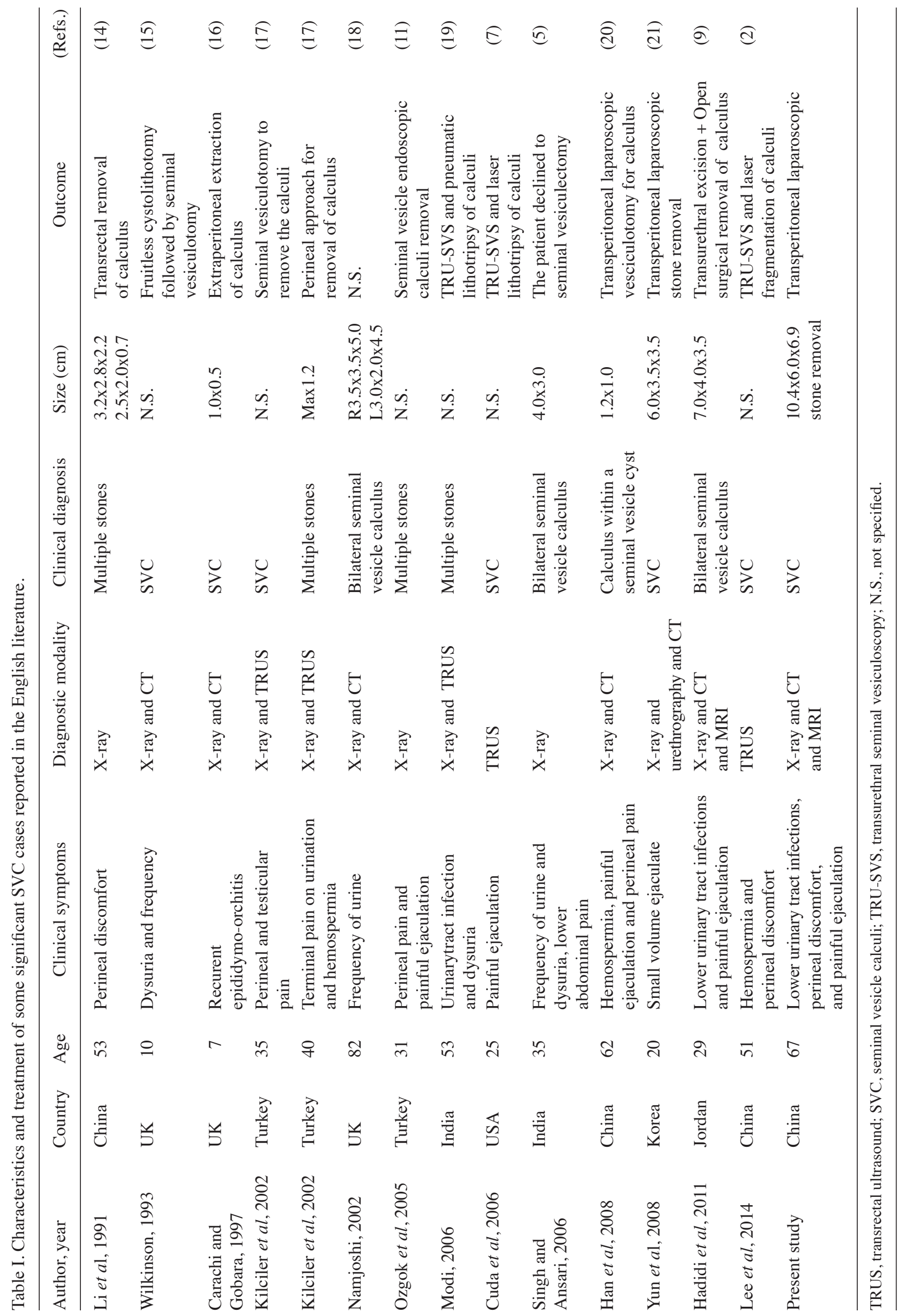




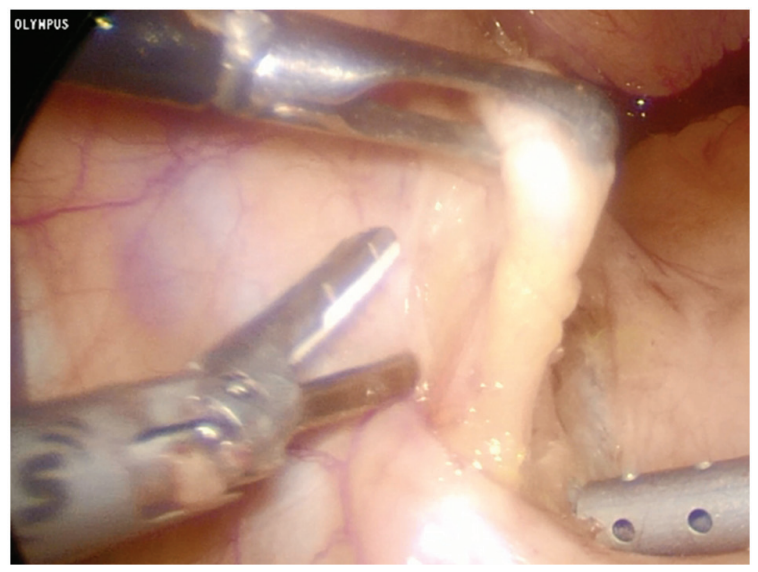

Figure 4. The left vas deferens was used as a guide for the removal of the seminal vesicle.

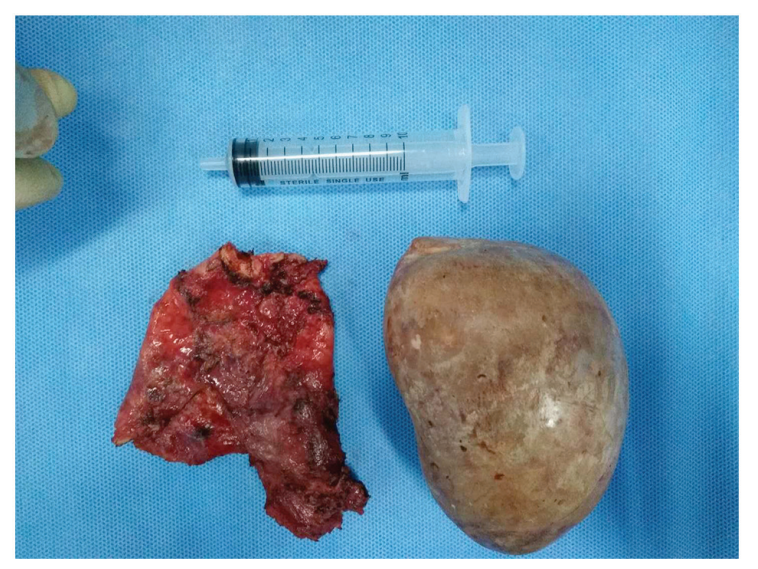

Figure 5. Image of the light brown-colored, round and smooth calculus and the dilated left seminal vesicle wall extracted.

tract obstruction, anatomic anomaly, seminal vesicle cysts or reflux (2). In addition, $16.2 \%$ of patients with hemospermia have SVC (3). Although certain patients are asymptomatic, frequent lower urinary tract infections, haematospermia and ejaculatory pain, perineum or testicular pain are common (4). Furthermore, a series of lower urinary tract symptoms have been associated with frequency and urgency to urinate, dysuria and urinary tract infections $(1,4)$. The most common symptom/complaint of a patient seeking medical care is hematospermia and ejaculation pain, and this may subside if left untreated. The patient of the present study had a history of numerous years of recurrent urinary tract infections. The major symptoms included urgency and increased frequency of urination, lower abdominal and perineal pain and occasionally hematuria. The patient's self-administered antibiotics improved his symptoms, which may in part be the reason why he did not seek hospital care for treatment previously.

The diagnosis of SVC usually involves a combination of clinical symptoms, rectal digital examination and X-rays (5). MRI are more sensitive compared with transrectal ultrasound and CT in the diagnosis of SVC (6,7). SVC frequently requires surgical treatment. In previous case reports, transurethral, suprapubic, transrectal or perineal procedures were applied for the treatment of stones (8). For large calculus, open supra-pubic incision and cystotomy is usually selected (9). Transurethral seminal vesiculoscopy and laparoscopic approach are currently in use as effective treatments for SVC. Table I lists the characteristics and treatments of certain typical cases that have been reported in the English literature.

The first seminal vesiculoscopy was reported in 1996 (10) and the first endoscopic lithotripsy was reported in 2005 (11). Subsequent studies have suggested that transurethral seminal vesiculoscopy is relatively simple to perform, is associated with a rapid post-operative recovery, to have fewer complications than transurethral resection of ejaculatory ducts and to preserve the normal structure of seminal vesicles and ejaculatory ducts in the treatment of seminal vesicle disease (12). It is particularly advantageous when dealing with small stones of seminal vesicles. Transperitoneal laparoscopy is considered a safe and thorough treatment for large stones (1). To the best of our knowledge, the SVC of the present case is the largest reported in the English literature and laparoscopic surgery was selected for treatment. The patient was 67 years old, infertile, had low requirements for sexual and reproductive functions and decided not to retain the seminal vesicle at the pre-operative consultation

This patient had a history of hypospadias, cryptorchidism, and azoospermic, and a similar medical history was observed in another case (9). Both of them were infertile. There is currently no clear evidence of an association between SVC and hypospadias (9). Hypoplasia, agenesis and congenital cysts of the seminal vesicles have been suggested to be linked to cryptorchidism (13). Anomalies of the seminal vesicles can affect patients of any age, especially after sexual maturity it become more obvious (9). Based on the current health awareness of the general population and medical improvements, the number of patients with SVC will increase; however, large seminal calculi may be difficult to detect. The patient of the present study was not the first case of large SVC accompanied with hypospadias and cryptorchidism reported. Further research is warranted to determine whether there may be a link with chromosome variations.

\section{Acknowledgements}

Not applicable.

\section{Funding}

No funding was received.

\section{Availability of data and materials}

All data generated or analyzed during this study are included in this published article.

\section{Authors' contributions}

HH conceived and supervised the study. HY, YW and JG acquired the data. PW, XJ and DT retrieved and reviewed the literature, analyzed the results and critically revised the manuscript for intellectual content. HY drafted the manuscript. $\mathrm{HH}$ and HY prepared figures and tables. All authors have read and approved the final manuscript. 


\section{Ethics approval and consent to participate}

The present study was approved by the Ethics Committee of the Department of Urology, The Second Hospital of Tianjin Medical University (Tianjin, China).

\section{Patient consent for publication}

Informed consent for publication was obtained from the patient.

\section{Competing interests}

The authors declare that they have no competing interests.

\section{References}

1. Christodoulidou M, Parnham A and Nigam R: Diagnosis and management of symptomatic seminal vesicle calculi. Scand J Urol 51: 1-8, 2017.

2. Lee TH, Juan YS, Jang MY, Wang HS, Shen JT, Lee TH, Juan YS, Jang MY, Wang HS and Shen JT: Successful seminal vesiculoscopic lithotripsy of seminal vesicle stone: A case report and literature review. Urolog Sci 25: 134-146, 2014.

3. Yang SC, Rha KH, Byon SK and Kim JH: Transutricular seminal vesiculoscopy. J Endourol 16: 343-345, 2002.

4. Corriere JN Jr: Painful ejaculation due to seminal vesicle calculi. J Urol 157: 626, 1997.

5. Singh I and Ansari M: Idiopathic bilateral giant seminal vesicle calculi and calcification of the male ejaculatory system: Current review of diagnosis and management. Indian J Surg 68: 38-40, 2006.

6. Cho IR, Lee MS, Rha KH, Hong SJ, Park SS and Kim MJ: Magnetic resonance imaging in hemospermia. J Urol 157: 258-262, 1997.

7. Cuda SP, Brand TC, Thibault GP and Stack RS: Case report: Endoscopic laser lithotripsy of seminal-vesicle stones. J Endourol 20: 916-918, 2006.

8. Schwartz BF: Stones of the urethra, prostate, seminal vesicle, bladder, and encrusted foreign bodies. In: Urinary Stone Disease. Stoller ML and Meng MV (eds). Humana Press, New Jersey, pp661-681, 2007.
9. Hadidi M, Hadidy A, Alrabadi AF, Ahdul-Wahab AD and Murshidi MM: Bilateral very large calcium oxalate stones in the seminal vesicles: Case report and literature review. Urol Res 39: 509-513, 2011.

10. Shimada $M$ and Yoshida $\mathrm{H}$ : Ex vivo ultrathin endoscopy of the seminal vesicles. J Urol 156: 1388-1390, 1996.

11. Ozgok Y, Kilciler M, Aydur E, Saglam M, Irkilata HC and Erduran D: Endoscopic seminal vesicle stone removal. Urology 65: 591, 2005.

12. Han CH, Liang Q, Dong BZ, Hao L, Fan T, Zhang JJ, Zhang WD, Chen B, Qiu XZ, Zhou XJ and Pei CS: The transurethral seminal vesiculoscopy in the diagnosis and treatment of the seminal vesicle disease. Cell Biochem Biophys 66: 851-853, 2013.

13. King BF, Hattery RR, Lieber MM, Williamson B Jr, Hartman GW and Berquist TH: Seminal vesicle imaging. Radiographics: A review publication of the Radiological Society of North America, Inc. 9, 653-676, 1989.

14. Li YK: Diagnosis and management of large seminal vesicle stones. Br J Urol 68: 322-323, 1991.

15. Wilkinson AG: Case report: Calculus in the seminal vesicle. Pediatr Radiol 23: 327, 1993.

16. Carachi R and Gobara D: Recurrent epididymo-orchitis in a child secondary to a stone in the seminal vesicle. Br J Urol 79: 997, 1997.

17. Kilciler M, Saglam M, Ozgok Y, Somuncu I, Erduran D and Harmankaya C: Giant seminal vesicle stones. Report of two cases. Urol Int 69: 250-251, 2002.

18. Namjoshi SP: Large bilateral star-shaped calculi in the seminal vesicles. J Postgrad Med 48: 122-123, 2002.

19. Modi PR: Case report: Endoscopic management of seminal vesicle stones with cutaneous fistula. J Endourol 20: 432-435, 2006.

20. Han P, Yang YR, Zhang XY and Wei Q: Laparoscopic treatment of a calcium fluorophosphate stone within a seminal vesicle cyst. Asian J Androl 10: 337-340, 2008.

21. Yun SJ, Kim TH, Kwon WA, Kim YJ, Lee SC and Kim WJ: A large stone in the dilated left seminal vesicle: Laparoscopic removal and partial seminal vesiculectomy. Korean J Urol 49: 656-658, 2008.

(i) $\Theta$ This work is licensed under a Creative Commons Attribution-NonCommercial-NoDerivatives 4.0 International (CC BY-NC-ND 4.0) License. 